\title{
The Thermal Statistics of Quasi-Probabilities' Analogs in Phase Space
}

\author{
F. Pennini, ${ }^{1,2}$ A. Plastino, ${ }^{3}$ and M. C. Rocca $^{3}$ \\ ${ }^{1}$ Departamento de Física, Facultad de Ciencias Exactas y Naturales, Universidad Nacional de La Pampa, Avenida Peru 151, \\ Santa Rosa, 6300 La Pampa, Argentina \\ ${ }^{2}$ Departamento de Física, Universidad Católica del Norte, Avenida Angamos 0610, 1270709 Antofagasta, Chile \\ ${ }^{3}$ Instituto de Física La Plata-CCT-CONICET, Universidad Nacional de La Plata, C.C. 727, 1900 La Plata, Argentina
}

Correspondence should be addressed to F. Pennini; fpennini@ucn.cl

Received 14 May 2015; Revised 30 July 2015; Accepted 23 August 2015

Academic Editor: Klaus Kirsten

Copyright ( 2015 F. Pennini et al. This is an open access article distributed under the Creative Commons Attribution License, which permits unrestricted use, distribution, and reproduction in any medium, provided the original work is properly cited.

We focus attention upon the thermal statistics of the classical analogs of quasi-probabilities (QP) in phase space for the important case of quadratic Hamiltonians. We consider the three more important OPs: Wigner's, $P$-, and Husimi's. We show that, for all of them, the ensuing semiclassical entropy is a function only of the fluctuation product $\Delta x \Delta p$. We ascertain that the semiclassical analog of $P$-distribution seems to become unphysical at very low temperatures. The behavior of several other information quantifiers reconfirms such an assertion in manifold ways. We also examine the behavior of the statistical complexity and of thermal quantities like the specific heat.

\section{Introduction}

A quasi-probability distribution is a mathematical construction that resembles a probability distribution but does not necessarily fulfill some of Kolmogorov's axioms for probabilities [1]. Quasi-probabilities exhibit general features of ordinary probabilities. Most importantly, they yield expectation values with respect to the weights of the distribution. However, they disobey the third probability postulate [1] in the sense that regions integrated under them do not represent probabilities of mutually exclusive states. Some quasiprobability distributions exhibit zones of negative probability density. This kind of distributions often arises in the study of quantum mechanics when discussed in a phase space representation of frequent use in quantum optics, timefrequency analysis, and so forth.

One usually considers a density operator $\hat{\rho}$ defined with respect to a complete orthonormal basis and shows that it can always be written in a diagonal manner, provided that an overcomplete basis is at hand [2]. This is the case of coherent states $|\alpha\rangle$ [3], for which [2]

$$
\widehat{\rho}=\int \frac{d^{2} \alpha}{\pi} P\left(\alpha, \alpha^{*}\right)|\alpha\rangle\langle\alpha| .
$$

We have $d^{2} \alpha / \pi=d x d p / 2 \pi \hbar$, with $x$ and $p$ being phase space variables. Coherent states, right eigenstates of the annihilation operator $\widehat{a}$, serve as the overcomplete basis in such a build-up $[2,3]$.

There exists a family of different representations, each connected to a different ordering of the creation and destruction operators $\hat{a}$ and $\hat{a}^{\dagger}$. Historically, the first of these is the Wigner quasi-probability distribution $W$ [4], related to symmetric operator ordering. In quantum optics the particle number operator is naturally expressed in normal order and in the pertinent scenario the associated representation of the phase space distribution is the Glauber-Sudarshan $P$ one [3]. In addition to $W$ and $P$, one may find many other quasi-probability distributions emerging in alternative representations of the phase space distribution [5]. A quite popular representation is the Husimi $Q$ one [6-9], used when operators are in antinormal order. We emphasize that we work here with classical analogs of $W, P$, and $Q$. As stated, we will specialize things to the three $f$-functions associated to a Harmonic Oscillator (HO) of angular frequency $\omega$. In such a scenario the three (classical analog) functions that we call for convenience $f_{P}, f_{\mathrm{Q}}$, and $f_{W}$ are just Gaussians. The pertinent treatment becomes wholly analytical. 
1.1. Our Goal. In this paper, we wish to apply semiclassical information theory tools associated with these analog $P$, $Q$, and $W$ representations (for quadratic Hamiltonians) in order to describe the associated thermal semiclassical features. The idea is to gain physical insight from the application of different information quantifiers to classical analogs of quasiprobability distributions. It will be seen that useful insights are in this way gained. WE will discover that, out of the three functions, only $f_{Q}$ and $f_{W}$ are sensible analogs, while $f_{P}$ exhibits problems if the temperature is low enough.

We insist that, in this paper, we will regard quasiprobabilities as semiclassical distributions in phase space, analogs of the quantum quasi-probabilistic distributions, and try to ascertain what physical features they are able to describe at such semiclassical level. One has $[10,11]$

$$
\begin{aligned}
& f_{P}=\gamma_{P} e^{-\gamma_{P}|\alpha|^{2}}, \gamma_{P}=e^{\beta \hbar \omega}-1 \quad(P \text {-function }), \\
& f_{\mathrm{Q}}=\gamma_{\mathrm{Q}} e^{-\gamma_{\mathrm{Q}}|\alpha|^{2}}, \gamma_{\mathrm{Q}}=1-e^{-\beta \hbar \omega} \quad(\mathrm{Q} \text {-function }), \\
& f_{W}=\gamma_{W} e^{-\gamma_{W}|\alpha|^{2}}, \\
& \gamma_{W}=2 \tanh \left(\frac{\beta \hbar \omega}{2}\right) \quad(W \text {-function }),
\end{aligned}
$$

where $\beta=1 / k_{B} T, k_{B}$ is the Boltzmann constant, and $T$ is the temperature. They will be used as semiclassical statistical weight functions. Since ours is not a quantum approach, the ordering of HO-creation and destruction operators $\widehat{a}$ and $\hat{a}^{\dagger}$ plays no role whatsoever.

1.2. Historic Considerations and Organization. The thermodynamics properties associated with coherent states have been the subject of much interest. See, for instance, $[12,13]$. Notice that $\mathrm{HO}$ is a really relevant system that yields useful insights of wide impact. Indeed, $\mathrm{HO}$ constitutes much more than a mere example. It is of special relevance for bosonic or fermionic atoms contained in magnetic traps [14-16] and for system that exhibits an equidistant level spacing in the vicinity of the ground state, like nuclei or Luttinger liquids.

For thermal states, the Gaussian $\mathrm{HO}$-quantum phase spaces distributions are known in the literature for applications in quantum optics.

This paper is organized as follows. Section 2 refers to different information quantifiers in a phase space representation for Gaussian distributions. In Section 3 we calculate the classical analog Fano factor. Features of the fluctuations are analyzed in Section 4. Additionally, we discuss the notion of linear entropy. Finally, some conclusions are drawn in Section 5 .

\section{Semiclassical Information Quantifiers}

Consider a general normalized gaussian distribution in phase space

$$
f(\alpha)=\gamma e^{-\gamma|\alpha|^{2}}
$$

whose normalized variance is $1 / \gamma$ with $\gamma$ taking values $\gamma_{P}$, $\gamma_{Q}$, and $\gamma_{W}$. We discuss next, in these terms, some important information theory quantifiers.

2.1. Shift-Invariant Fisher's Information Measure. The information quantifier Fisher's information measure, specialized for families of shift-invariant distributions, which do not change shape under translations, is $[17,18]$

$$
I=\int d x f(x)\left(\frac{\partial \ln f(x)}{\partial x}\right)^{2},
$$

and, in phase space, it adopts the appearance [19]

$$
I=\frac{1}{4} \int \frac{d^{2} \alpha}{\pi} f(\alpha)\left(\frac{\partial \ln f(\alpha)}{\partial|\alpha|}\right)^{2}
$$

such that considering $f(\alpha)$ given by (3) we get $I=\gamma$, whose specific values are $\gamma_{P}, \gamma_{Q}$, and $\gamma_{W}$ for the three functions $f_{P}$, $f_{Q}$, and $f_{W}$. The behavior of these quantities is displayed in Figure 1. The solid line is the case $P$, the dashed one is the Wigner one, and the dotted curve is assigned to the Husimi case. Now, it is known that in the present scenario the maximum attainable value for $I$ equals 2 [19]. The $P$-result violates this restriction at low temperatures more precisely at

$$
T<T_{\text {crit }}=\frac{\left(\hbar \omega / k_{B}\right)}{\ln 3} \approx 0.91023 \frac{\hbar \omega}{k_{B}},
$$

with $T$ being expressed in $\left(\hbar \omega / k_{B}\right)$-units.

2.2. Logarithmic Entropy S. The logarithmic Boltzmann's information measure for the the probability distribution (3) is

$$
S=-\int \frac{d^{2} \alpha}{\pi} f(\alpha) \ln f(\alpha)=1-\ln \gamma
$$

so that it acquires the particular values

$$
\begin{aligned}
& S_{P}=1-\ln \left(e^{\beta \hbar \omega}-1\right), \\
& S_{\mathrm{Q}}=1-\ln \left(1-e^{-\beta \hbar \omega}\right), \\
& S_{W}=1-\ln \left(2 \tanh \left(\frac{\beta \hbar \omega}{2}\right)\right),
\end{aligned}
$$

for, respectively, the distributions $f_{P}, f_{Q}$, and $f_{W}$. These entropies are plotted in Figure 2. Notice that $S_{P}<0$ for $T<\hbar \omega /\left(k_{B} \ln (1+e)\right) \approx 0.76\left(\hbar \omega / k_{B}\right)<T_{\text {crit }}$. Negative classical entropies are well known. As an example, one can cite [20].

Table 1 lists a set of critical temperatures $T_{\text {crit }}$ for typical electromagnetic (EM) waves.

We see from Table 1 that serious anomalies are detected for $P$-distribution in the case of radio waves of high frequency. $P$ becomes negative, which is absurd, for rather high temperatures, where one expects classical physics to reign. Accordingly, one concludes that quasi-probabilities do not exhibit a sensible classical limit in $P$-case contrary to what happens in both $W$ and $Q$ ones. 
TABLE 1: Critical temperatures $T_{\text {crit }}$ for typical radio waves, with $h / k_{B}=4.79910^{-11}$ Kelvin per second.

\begin{tabular}{lcc}
\hline & Frequency $(\nu)$ & Critical temperatures $\left({ }^{\circ} \mathrm{K}\right)$ \\
\hline Extremely low frequency (ELF) & $3-30 \mathrm{~Hz}$ & $1.439710^{-10}-1.439710^{-9}$ \\
Super low frequency (SLF) & $30-300 \mathrm{~Hz}$ & $1.439710^{-9}-1.439710^{-8}$ \\
Ultra low frequency (ULF) & $300-3000 \mathrm{~Hz}$ & $1.439710^{-8}-1.439710^{-7}$ \\
Very low frequency (VLF) & $3-30 \mathrm{kHz}$ & $1.439710^{-7}-1.439710^{-6}$ \\
Low frequency (LF) & $30-300 \mathrm{kHz}$ & $1.439710^{-6}-1.439710^{-5}$ \\
Medium frequency (MF) & $300 \mathrm{KHz}-3 \mathrm{MHz}$ & $1.439710^{-5}-1.439710^{-4}$ \\
High frequency (HF) & $3-30 \mathrm{MHz}$ & $1.439710^{-4}-1.439710^{-3}$ \\
Very high frequency (VHF) & $30-300 \mathrm{MHz}$ & $1.439710^{-3}-1.439710^{-2}$ \\
Ultra high frequency (UHF) & $300 \mathrm{MHz}-3 \mathrm{GHz}$ & $1.439710^{-2}-1.439710^{-1}$ \\
Super high frequency (SHF) & $3-30 \mathrm{GHz}$ & $1.439710^{-1}-1.4397$ \\
Extremely high frequency (EHF) & $30-300 \mathrm{GHz}$ & $1.4397-14.397$ \\
Tremendously high frequency (THF) & $300 \mathrm{GHz}-3000 \mathrm{GHz}$ & $14.397-143.97$ \\
\hline
\end{tabular}

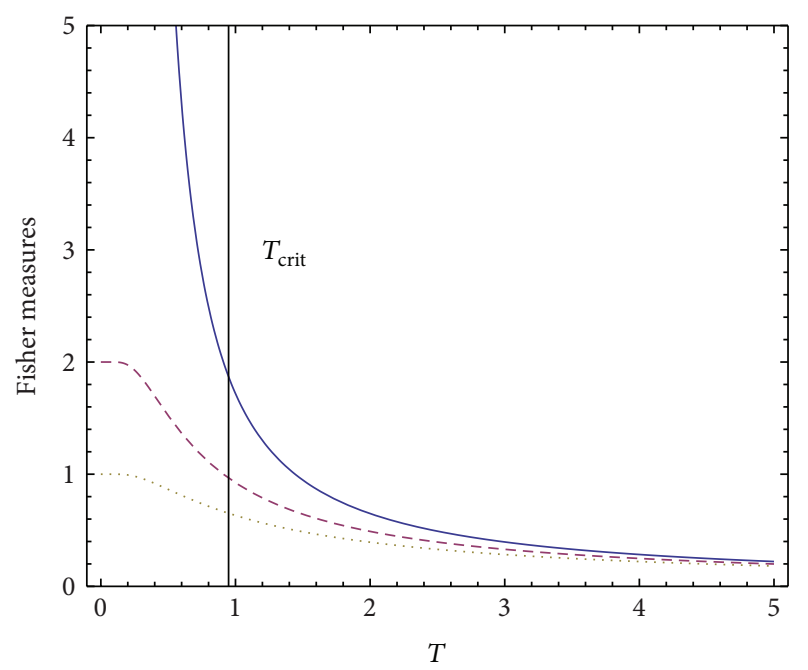

FIGURE 1: Fisher measure versus temperature $T$ expressed in $\left(\hbar \omega / k_{B}\right)$-units. The solid line is the case $P$, the dashed one is the Wigner one, and the dotted curve is assigned to the Husimi instance. The vertical line represents the critical temperature $T_{\text {crit }}$.

2.3. Statistical Complexity. The statistical complexity $C$, according to López-Ruiz et al. [21], is a suitable product of two quantifiers such that $C$ becomes minimal at the extreme situations of perfect order or total randomness. Instead of using the prescription of [21], but without violating its spirit, we will take one of these two quantifiers to be Fisher's measure and take the other to be an entropic form, since it is well known that the two behave in opposite manner [22]. Thus

$$
C=S I=\gamma(1-\ln \gamma),
$$

which vanishes for perfect order or total randomness. For each particular case, we explicitly have

$$
\begin{aligned}
C_{P} & =\left(e^{\beta \hbar \omega}-1\right)\left[1-\ln \left(e^{\beta \hbar \omega}-1\right)\right], \\
C_{Q} & =\left(1-e^{-\beta \hbar \omega}\right)\left[1-\ln \left(1-e^{-\beta \hbar \omega}\right)\right], \\
C_{W} & =2 \tanh \left(\frac{\beta \hbar \omega}{2}\right)\left[1-\ln \left(2 \tanh \left(\frac{\beta \hbar \omega}{2}\right)\right)\right],
\end{aligned}
$$

for, respectively, the distributions $f_{P}, f_{Q}$, and $f_{W}$. The maximum of the statistical complexity occurs when $\gamma=1$ and the associated temperature values are

$$
\begin{aligned}
& e^{\beta \hbar \omega}-1=1 \Longrightarrow T=\frac{\hbar \omega}{k_{B}} \ln 2>T_{\text {crit }} \\
& \text { for the } f_{P} \text {-function, } \\
& 1-e^{-\beta \hbar \omega}=1 \Longrightarrow T=0 \quad \text { for the } f_{Q} \text {-function, } \\
& 2 \tanh \left(\frac{\beta \hbar \omega}{2}\right)=1 \Longrightarrow T=\frac{\hbar \omega}{2 k_{B}} \arctan \left(\frac{1}{2}\right) \\
& \text { for the } f_{W} \text {-function. }
\end{aligned}
$$

The statistical complexity $C$ is plotted in Figure 3.

2.4. Linear Entropy. Another interesting information quantifier is that of the Manfredi-Feix entropy [23], derived from the phase space Tsallis $(q=2)$ entropy [24]. In quantum information this form is referred to as the linear entropy [25]. It reads

$$
\begin{aligned}
& S_{l}=1-\int \frac{d^{2} \alpha}{\pi} f^{2}(\alpha)=1-\mathscr{J}, \\
& \mathscr{J}=\int \frac{d^{2} \alpha}{\pi} f^{2}(\alpha)=\frac{\gamma}{2} .
\end{aligned}
$$

Accordingly, we have

$$
S_{l}=1-\frac{\gamma}{2} ; \quad 0 \leq S_{l} \leq 1
$$

This is semiclassical result, which is valid for small $\gamma$. In particular,

$$
\begin{aligned}
& S_{l, P}=1-\frac{\gamma_{P}}{2}=1-\frac{e^{\beta \hbar \omega}-1}{2}, \\
& S_{l, Q}=1-\frac{\gamma_{Q}}{2}=1-\frac{1-e^{-\beta \hbar \omega}}{2}, \\
& S_{l, W}=1-\frac{\gamma_{W}}{2}=1-\frac{2 \tanh (\beta \hbar \omega / 2)}{2} .
\end{aligned}
$$




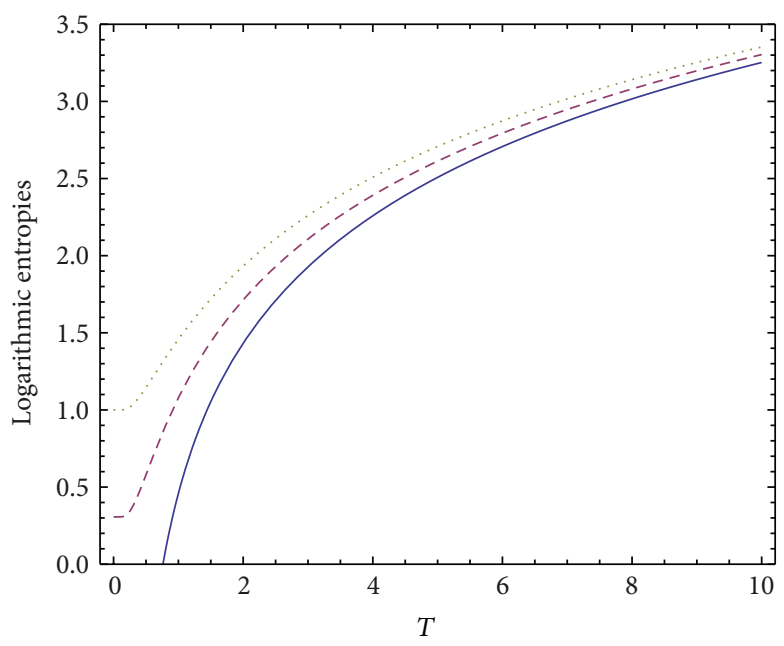

(a)

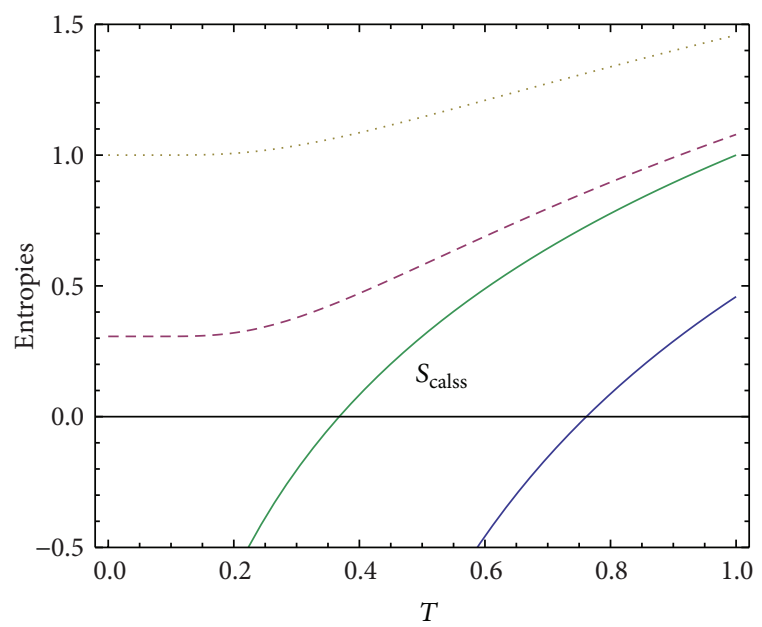

(b)

FIgURE 2: Left: logarithmic entropies, $S_{P}, S_{Q}$, and $S_{W}$, as a function of the temperature $T$ in $\left(\hbar \omega / k_{B}\right)$-units. The solid line is the case $P$, the dashed one is the Wigner one, and the dotted curve is assigned to the Husimi instance. Right: zoom of the logarithmic entropies as a function of temperature $T$ in $\left(\hbar \omega / k_{B}\right)$-units. Negative values of $S_{P}$ occur below $T=\hbar \omega /\left(k_{B} \ln (1+e)\right)$, with the critical temperature $<T_{\text {crit }}$. Remaining details are similar to those of left figure. We have added the classical entropy of the harmonic oscillator $S_{\text {class }}=1-\ln (\beta \hbar \omega)$.

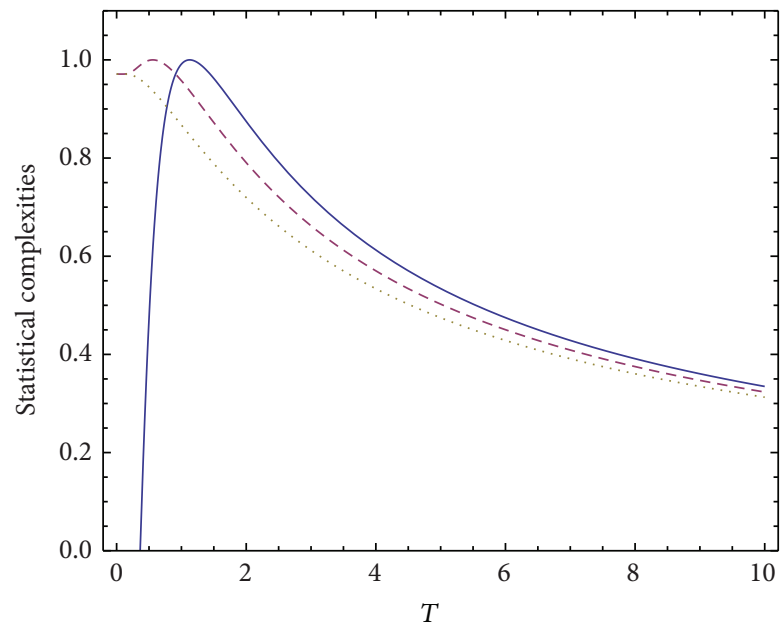

Figure 3: Complexities $C_{P}, C_{Q}$, and $C_{W}$ versus the temperature $T$ in $\left(\hbar \omega / k_{B}\right)$-units. The solid line is the case $P$, the dashed one is the Wigner one, and the dotted curve is assigned to the Husimi instance.

Note that in the $P$-instance the linear entropy becomes negative once again for $T<T_{\text {crit }}$. Contrary to what happens for the logarithmic entropy, the linear one can vanish in $W$ representation. The lineal entropies are plotted in Figure 4.

The ensuing statistical complexity that uses $S_{l}$ becomes

$$
C_{l}=S_{l} I=I\left(1-\frac{I}{2}\right)=\gamma\left(1-\frac{\gamma}{2}\right)
$$

vanishing for both $\gamma=0$ and $\gamma=2$, the extreme values of $\gamma$-physical range (we showed above that $\gamma$ cannot exceed 2 without violating uncertainty restrictions).

\section{Fano Factor's Classical Analog}

In general, the Fano factor is the coefficient of dispersion of the probability distribution $p(y)$, which is defined as [26]

$$
\mathscr{F}=\frac{\Delta y^{2}}{\langle y\rangle},
$$

where $\Delta y^{2}=\left\langle y^{2}\right\rangle-\langle y\rangle^{2}$ is the variance and $\langle x\rangle$ is the mean of a random process $y$.

If $p(y)$ is a Poisson distribution then one sees that the pertinent Fano factor becomes unity $(\mathscr{F}=1)[10,27]$. We remind the reader of two situations:

(1) For $\mathscr{F}<1$, sub-Poissonian processes occur.

(2) For $\mathscr{F}>1$, the process is super-Poissonian.

For our Gaussian distribution (3), if one sets now $y=|\alpha|^{2}$, one has the classical Fano analog

$$
\mathscr{F}=\frac{\left\langle|\alpha|^{4}\right\rangle_{f}-\left\langle|\alpha|^{2}\right\rangle_{f}^{2}}{\left\langle|\alpha|^{2}\right\rangle_{f}},
$$

where the expectation value of the function $\mathscr{A}(\alpha)$ is calculated as

$$
\langle\mathscr{A}\rangle_{f}=\int \frac{d^{2} \alpha}{\pi} f(\alpha) \mathscr{A}(\alpha),
$$

indicating that $f(\alpha)$ is the statistical weight function. Thus, after computing the mean values involved in (22) by taking into account definition (23), the Fano factor becomes

$$
\mathscr{F}=\frac{1}{\gamma}=\frac{1}{I},
$$




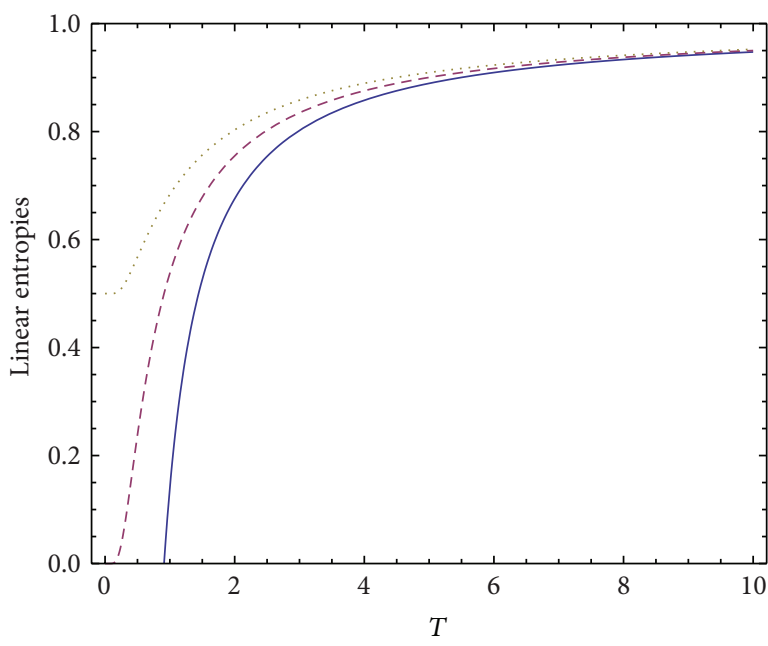

(a)

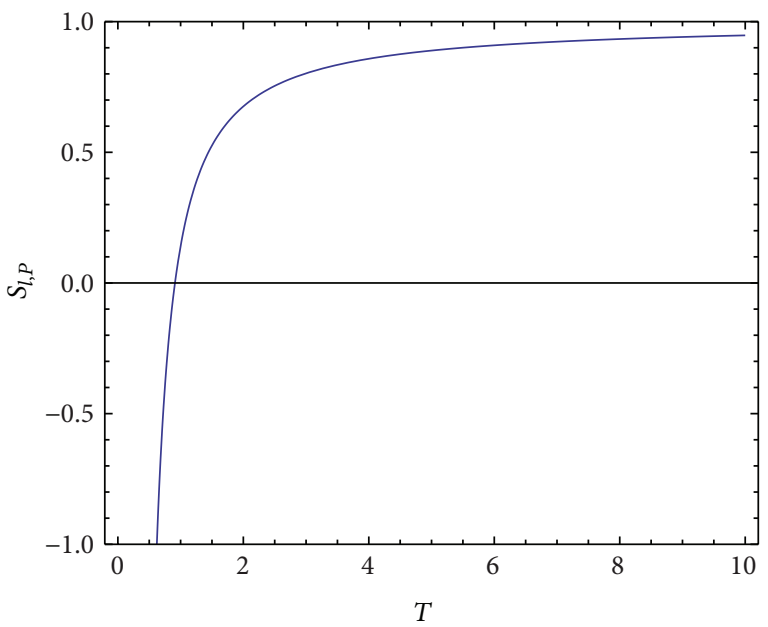

(b)

FIgURE 4: Left: linear entropies $S_{l, P}, S_{l, Q}$, and $S_{l, W}$ versus the temperature $T$ in $\left(\hbar \omega / k_{B}\right)$-units. The solid line is the case $P$, the dashed one is the Wigner one, and the dotted curve is assigned to the Husimi instance. Right: $S_{l, P}$ as a function of the temperature $T$ in $\left(\hbar \omega / k_{B}\right)$-units. We note that the linear entropy $S_{l, P}$ is negative below the $T$ value $t_{\text {crit }}=T_{\text {crit }} k_{B} / \hbar \omega=\ln 3$.

which, for a Gaussian distribution, links the Fano factor to the distribution's width and to Fisher's measure $I$. We are speaking of processes that are of a quantum nature and cannot take place in a classical environment. Thus, with reference to the critical temperature defined in (6), we have to deal with

$$
\mathscr{F}_{P}=\frac{1}{e^{\beta \hbar \omega}-1}\left(=1 \text { at } T=\frac{\hbar \omega}{k_{B} \ln 2}>T_{\text {crit }}\right)
$$

for the $f_{P}$ function,

$$
\mathscr{F}_{\mathrm{Q}}=\frac{1}{1-e^{-\beta \hbar \omega}}(=1 \text { at } T=0)
$$

for the $f_{\mathrm{Q}}$ function,

$$
\mathscr{F}_{W}=\frac{1}{2 \tanh (\beta \hbar \omega / 2)}\left(=1 \text { at } T=T_{\text {crit }}\right)
$$

for the $f_{W}$ function.

The $f_{\mathrm{Q}}$-case reaches the super- to sub-Poissonian transition only at $T=0$, while the other two cases reach it at finite temperatures.

\section{Fluctuations}

We start this section considering the classical Hamiltonian of the harmonic oscillator that reads

$$
\mathscr{H}(x, p)=\hbar \omega|\alpha|^{2},
$$

where $x$ and $p$ are phase space variables, $|\alpha|^{2}=x^{2} / 4 \sigma_{x}^{2}+$ $p^{2} / \sigma_{p}^{2}, \sigma_{x}^{2}=\hbar / 2 m \omega$, and $\sigma_{p}^{2}=\hbar m \omega / 2[28]$.
Using the definition of the mean value (23), from (26), we immediately find [29]

$$
\left\langle\frac{x^{2}}{2 \sigma_{x}^{2}}\right\rangle_{f}=\left\langle\frac{p^{2}}{2 \sigma_{p}^{2}}\right\rangle_{f}=\left\langle|\alpha|^{2}\right\rangle_{f},
$$

with

$$
\left\langle|\alpha|^{2}\right\rangle_{f}=\gamma \int \frac{d^{2} \alpha}{\pi} e^{-\gamma|\alpha|^{2}}|\alpha|^{2}=\frac{1}{\gamma},
$$

where $\langle x\rangle_{f}=\langle p\rangle_{f}=\langle\alpha\rangle_{f}=0$, while $\gamma$ takes the respective values $\gamma_{P}, \gamma_{\mathrm{Q}}$, and $\gamma_{W}$. The concomitant variances are $\Delta x^{2}=\left\langle x^{2}\right\rangle_{f}-\langle x\rangle_{f}^{2}=2 \sigma_{p}^{2} / \gamma$ and $\Delta p^{2}=\left\langle p^{2}\right\rangle_{f}-$ $\langle p\rangle_{f}^{2}=2 \sigma_{p}^{2} / \gamma$. Hence, for our general Gaussian distribution, one easily establishes that

$$
\mathcal{U}=\Delta x \Delta p=\frac{\hbar}{\gamma},
$$

which shows that $\gamma$ should be constrained by the restriction

$$
\gamma \leq 2 \text {, }
$$

if one wishes the inequality

$$
\Delta x \Delta p \geq \frac{\hbar}{2}
$$

to hold.

Specializing (29) for our three quasi-probability distributions yields

$$
\begin{aligned}
& \Delta x \Delta p=\frac{\hbar}{e^{\beta \hbar \omega}-1}, \text { for } f_{P} \text { function, } \\
& \Delta x \Delta p=\frac{\hbar}{1-e^{-\beta \hbar \omega}}, \text { for } f_{\mathrm{Q}} \text { function, } \\
& \Delta x \Delta p=\frac{\hbar}{2 \tanh (\beta \hbar \omega / 2)}, \text { for } f_{W} \text { function. }
\end{aligned}
$$


These fluctuations are plotted in Figure 5. The restriction (31) applied to the $P$-result entails that it holds if

$$
T \geq \frac{\hbar \omega}{\ln 3 k_{B}}=T_{\text {crit }} \approx 0.91023 \frac{\hbar \omega}{k_{B}} .
$$

Thus, the distribution $f_{P}$ seems again to become unphysical at temperatures lower than $T_{\text {crit }}$, for which (31) is violated. From (29), we have $\gamma=\hbar / U$. Accordingly, if we insert this into (7), the logarithmic entropy $S$ can be recast in $\mathcal{U}$-terms via the relation

$$
S=1-\ln \left(\frac{\hbar}{\Delta x \Delta p}\right)
$$

(also demonstrated in [30] to hold for the Wehrl entropy) which vanishes for

$$
\Delta x \Delta p=\frac{\hbar}{e} .
$$

In the $P$-instance this happens at

$$
T=0.71463 \frac{\hbar \omega}{k_{B}} .
$$

At this temperature Heisenberg's-like condition (31) is violated. $W$ and $Q$ distributions do not allow for such a circumstance. Actually, in the Wigner case, which is exact, the minimum $S$-value is attained at $\beta=\infty$, where

$$
S_{\text {min }}=1-\ln 2 \approx 0.306 .
$$

The uncertainty restriction (31) seems to impede the phase space entropy to vanish, a sort of quasi-quantum effect. It is clear then that, in phase space, the logarithmic entropy, by itself, is an uncertainty indicator, in agreement with the work, in other scenarios, of several authors (see, for instance, [31] and references therein).

Define now the participation ratio's analog as $[32,33]$

$$
m=\frac{1}{g}=\frac{2}{\gamma},
$$

where $\mathscr{F}$ is given by (17). This is an important quantity that measures the number of pure states entering the mixture determined by our general Gaussian probability distribution of amplitude $\gamma[32,33]$. We again encounter troubles with the $P$-distribution in this respect. It is immediately realized by looking at Figure 6 that, for fulfilling the obvious condition $m \geq 1$, one needs a temperature $T \geq T_{\text {crit }}$.

\section{Conclusions}

We have investigated here the thermal statistics of quasiprobabilities' analogs $f(\alpha)$ in phase space for the important case of quadratic Hamiltonians, focusing attention on the three more important instances, that is, those of Wigner, $P_{-}^{-}$, and Husimi distributions:

(i) We emphasized the fact that for all of them the semiclassical entropy is a function only of the fluctuation

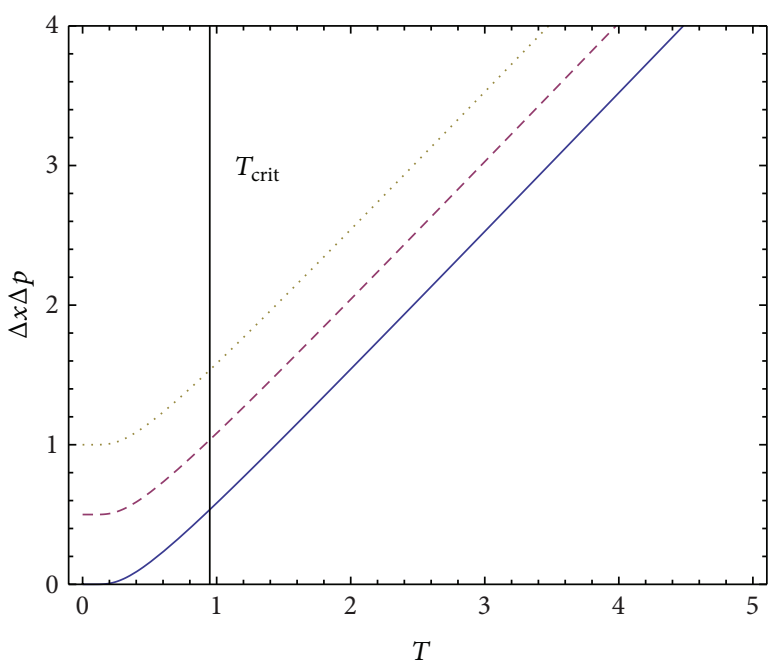

FIGURE 5: Fluctuations versus the temperature $T$ in $\left(\hbar \omega / k_{B}\right)$-units. The solid line is the case $P$, the dashed one is the Wigner one, and the dotted line is assigned to the Husimi instance.

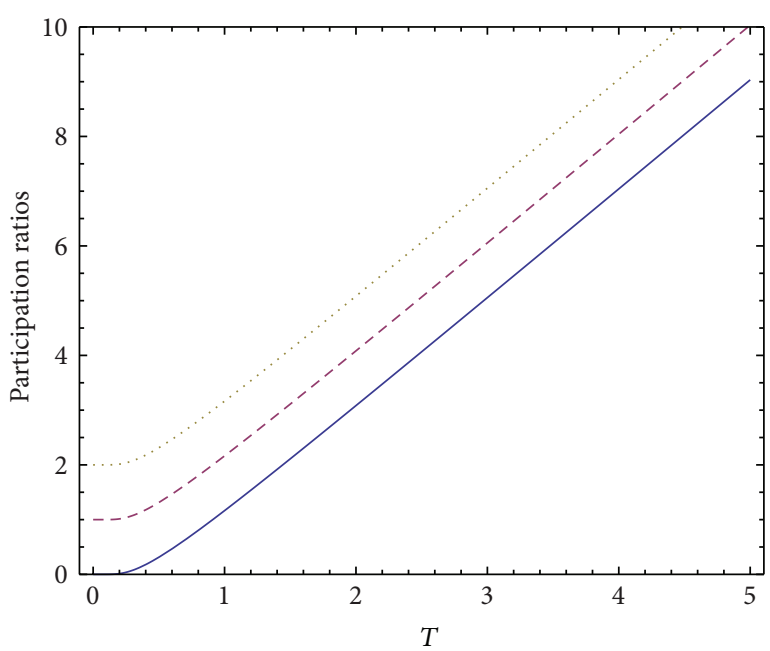

Figure 6: Participation ratio $m$ versus temperature $T$ in $\left(\hbar \omega / k_{B}\right)$ units. The solid line is the case $P$, the dashed one is the Wigner one, and the dotted curve is assigned to the Husimi instance.

product $\Delta x \Delta p$. This fact allows one to ascertain that the analog $P$-distribution seems to become unphysi$\mathrm{cal}$ at low enough temperatures, smaller than a critical value $T_{\text {crit }}$, because, in such an instance

(1) it would violate Heisenberg's-like principle in such a case. The behavior of other information quantifiers reconfirms such an assertion; that is,

(2) Fisher's measure exceeds its permissible maximum value $I=2$;

(3) the participation ratio becomes $<1$, which is impossible.

(ii) It is also clear then that semiclassical entropy, by itself, in phase space, looks like a kind of "uncertainty" indicator. 
(iii) We have determined the temperatures for which the statistical complexity becomes maximal as a signature of the well-known transition between classical and nonclassical states of light whose signature is the transition from super-Poissonian to sub-Poissonian distributions [34].

We have seen that the $P$-distribution in the case of radio waves of high frequency becomes negative, which is absurd, as the pertinent temperatures are rather high and thus one expects classical physics to reign. Accordingly, one concludes that quasi-probabilities do not exhibit a sensible classical limit in the $P$-case, contrary to what happens in both $W$ and $Q$ ones.

\section{Conflict of Interests}

The authors declare that there is no conflict of interests regarding the publication of this paper.

\section{Acknowledgments}

The authors were supported by Consejo Nacional de Investigaciones Científicas y Técnicas (CONICET), Argentina. Useful discussions with Professor R. Piasecki of Opole's University, Poland, are gratefully acknowledged.

\section{References}

[1] J. Von Plato, "Grundbegriffe der Wahrscheinlichkeitsrechnung," in Landmark Writings in Western Mathematics, I. Grattan-Guinness, Ed., pp. 960-969, Elsevier, Amsterdam, The Netherlands, 2005.

[2] E. C. Sudarshan, "Equivalence of semiclassical and quantum mechanical descriptions of statistical light beams," Physical Review Letters, vol. 10, pp. 277-279, 1963.

[3] R. J. Glauber, "Coherent and incoherent states of the radiation field," Physical Review, vol. 131, pp. 2766-2788, 1963.

[4] E. P. Wigner, "On the quantum correction for thermodynamic equilibrium," Physical Review, vol. 40, no. 5, pp. 749-759, 1932.

[5] F. Pennini and A. Plastino, "Smoothed Wigner-distributions, decoherence, and the temperature-dependence of the classicalquantum frontier," The European Physical Journal D, vol. 61, no. 1, pp. 241-247, 2011.

[6] K. Husimi, "Some formal properties of the density matrix," Proceedings of the Physico-Mathematical Society of Japan, vol. 22, pp. 264-283, 1940.

[7] S. S. Mizrahi, "Quantum mechanics in the Gaussian wavepacket phase space representation," Physica A, vol. 127, no. 1-2, pp. 241-264, 1984.

[8] S. S. Mizrahi, "Quantum mechanics in the Gaussian wavepacket phase space representation. II. Dynamics," Physica A. Statistical and Theoretical Physics, vol. 135, no. 1, pp. 237-250, 1986.

[9] S. S. Mizrahi, "Quantum mechanics in the Gaussian wavepacket phase space representation III: from phase space probability functions to wave-functions," Physica A, vol. 150, no. 3, pp. 541-554, 1988.

[10] M. O. Scully and M. S. Zubairy, Quantum Optics, Cambridge University Press, New York, NY, USA, 1997.
[11] W. P. Scheleich, Quantum Optics in Phase Space, Wiley, Berlin, Germany, 2001.

[12] J.-P. Gazeau, Coherent States in Quantum Physics, Wiley-VCH, Weinheim, Germany, 2009.

[13] E. H. Lieb, "The classical limit of quantum spin systems," Communications in Mathematical Physics, vol. 31, pp. 327-340, 1973.

[14] M. H. Anderson, J. R. Ensher, M. R. Matthews, C. E. Wieman, and E. A. Cornell, "Observation of Bose-Einstein condensation in a dilute atomic vapor," Science, vol. 269, no. 5221, pp. 198-201, 1995.

[15] K. B. Davis, M.-O. Mewes, M. R. Andrews et al., "Bose-Einstein condensation in a gas of sodium atoms," Physical Review Letters, vol. 75, no. 22, pp. 3969-3973, 1995.

[16] C. C. Bradley, C. A. Sackett, and R. G. Hulet, "Bose-Einstein condensation of lithium: observation of limited condensate number," Physical Review Letters, vol. 78, no. 6, pp. 985-989, 1997.

[17] B. R. Frieden and B. H. Soffer, "Lagrangians of physics and the game of Fisher-information transfer," Physical Review E, vol. 52, no. 3, pp. 2274-2286, 1995.

[18] M. J. W. Hall, "Quantum properties of classical Fisher information," Physical Review A, vol. 62, no. 1, Article ID 012107, 6 pages, 2000.

[19] F. Pennini and A. Plastino, "Fluctuations, entropic quantifiers and classical-quantum transition," Entropy, vol. 16, no. 3, pp. 1178-1190, 2014.

[20] E. H. Lieb, "Proof of an entropy conjecture of Wehrl," Communications in Mathematical Physics, vol. 62, no. 1, pp. 35-41, 1978.

[21] R. López-Ruiz, H. L. Mancini, and X. A. Calbet, "A statistical measure of complexity," Physics Letters A, vol. 209, no. 5-6, pp. 321-326, 1995.

[22] B. R. Frieden, Science from Fisher Information, Cambridge University Press, Cambridge, UK, 2nd edition, 2008.

[23] G. Manfredi and M. R. Feix, "Entropy and Wigner functions," Physical Review E, vol. 62, no. 4, pp. 4665-4674, 2000.

[24] C. Tsallis, Introduction to Nonextensive Statistical Mechanics, Springer, New York, NY, USA, 2008.

[25] P. Sadeghi, S. Khademi, and A. H. Darooneh, "Tsallis entropy in phase-space quantum mechanics," Physical Review A, vol. 86, no. 1, Article ID 012119, 2012.

[26] U. Fano, "Ionization yield of radiations. II. The fluctuations of the number of ions," Physical Review, vol. 72, no. 1, pp. 26-29, 1947.

[27] C. Brif and Y. Ben-Aryeh, "Subcoherent P-representation for non-classical photon states," Journal of the European Optical Society B: Quantum Optics, vol. 6, no. 5, pp. 391-396, 1994.

[28] R. K. Pathria, Statistical Mechanics, Pergamon Press, Exeter, UK, 1993.

[29] F. Pennini and A. Plastino, "Heisenberg-Fisher thermal uncertainty measure," Physical Review E, vol. 69, no. 5, Article ID 057101, 2004.

[30] A. Anderson and J. J. Halliwell, "Information-theoretic measure of uncertainty due to quantum and thermal fluctuations," Physical Review D, vol. 48, no. 6, pp. 2753-2765, 1993.

[31] I. Białynicki-Birula and J. Mycielski, "Uncertainty relations for information entropy in wave mechanics," Communications in Mathematical Physics, vol. 44, no. 2, pp. 129-132, 1975.

[32] J. Batle, A. R. Plastino, M. Casas, and A. Plastino, "Conditional q-entropies and quantum separability: a numerical exploration," Journal of Physics A:Mathematical and General, vol. 35, no. 48, pp. 10311-10324, 2002. 
[33] F. Pennini, A. Plastino, and G. L. Ferri, "Semiclassical information from deformed and escort information measures," Physica A, vol. 383, no. 2, pp. 782-796, 2007.

[34] X. T. Zou and L. Mandel, "Photon-antibunching and subPoissonian photon statistics," Physical Review A, vol. 41, no. 1, pp. 475-476, 1990. 


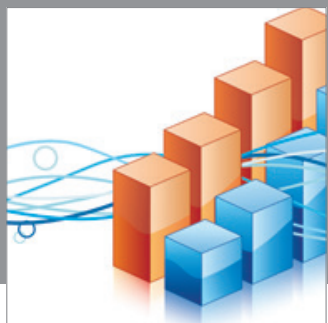

Advances in

Operations Research

mansans

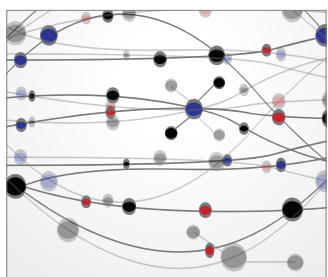

The Scientific World Journal
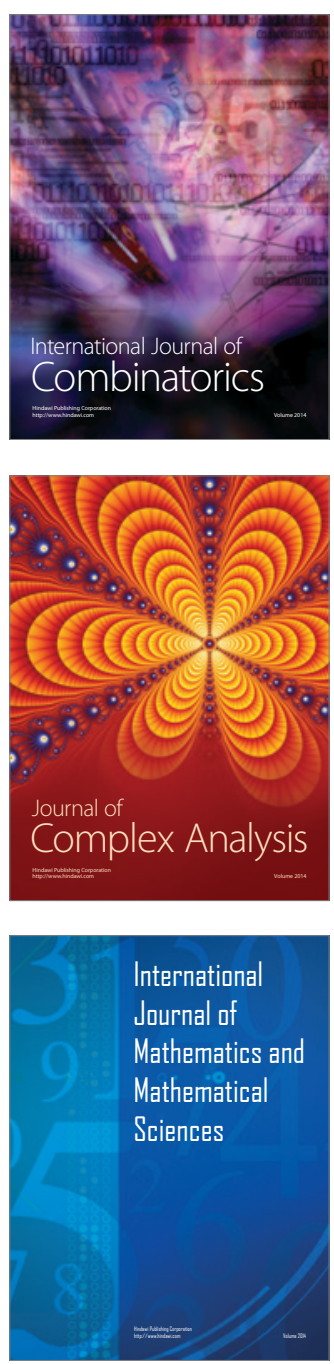
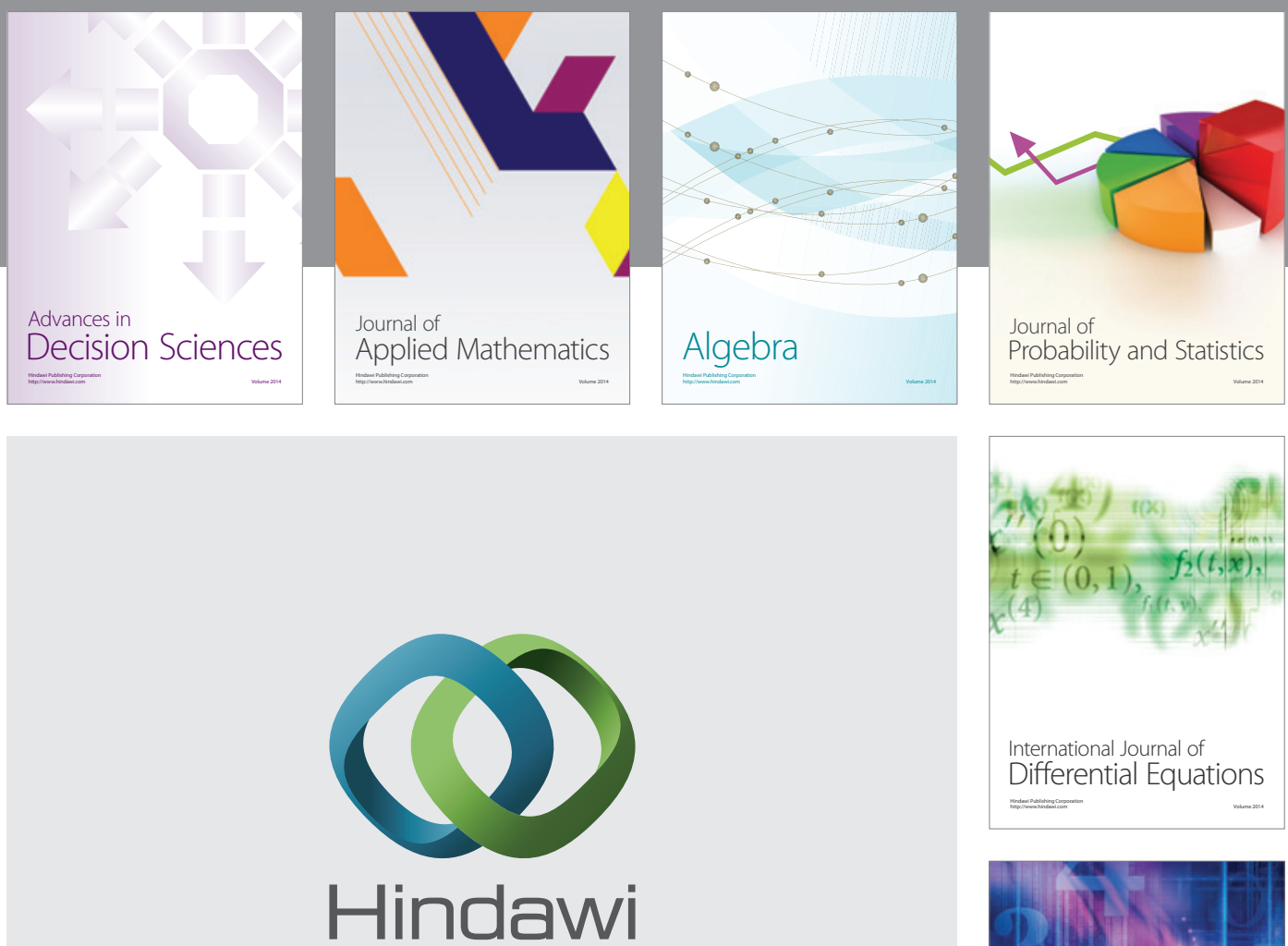

Submit your manuscripts at http://www.hindawi.com
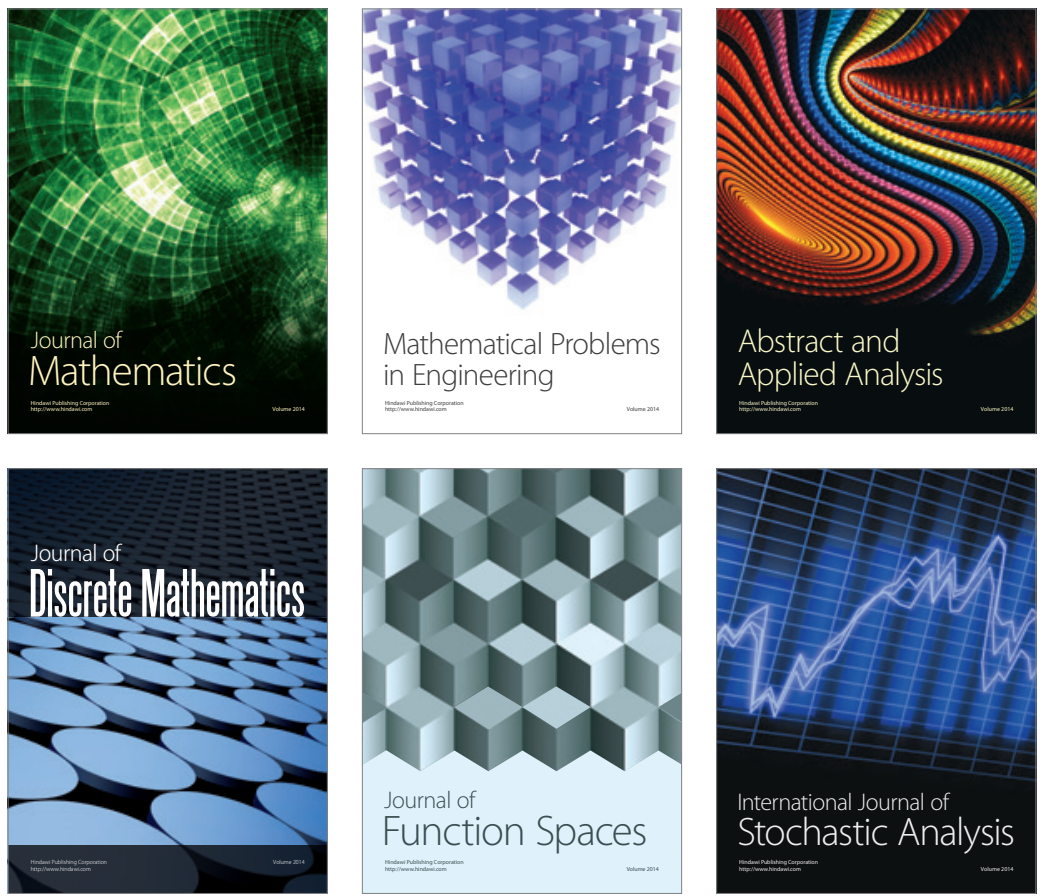

Journal of

Function Spaces

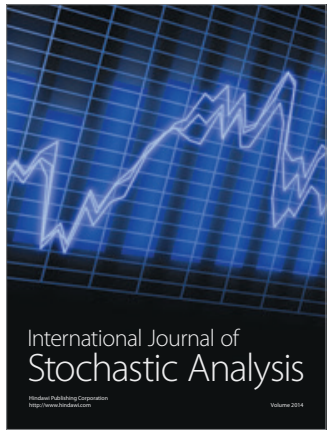

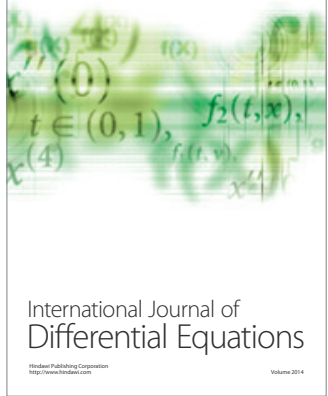
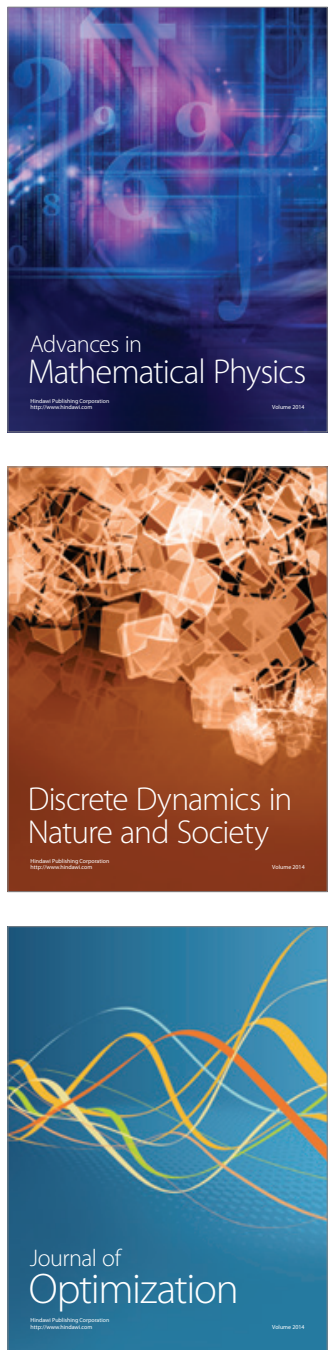